\title{
DYNAMICS OF DUST PARTICLES NEAR THE ROUND BELL SUCTION INLET
}

\author{
O. A. Averkova ${ }^{1}$, K. I. Logachev ${ }^{1}$, A. K. Logachev ${ }^{1}$, and E. I. Tolmacheva ${ }^{1}$ \\ ${ }^{1}$ Belgorod State Technological University named after V.G. Shukhov, \\ 308012 Belgorod, Russia, Kostukova str., 46 \\ e-mail: \{olga_19572004, kilogachev, artlogachev, lenka_110290\}@mail.ru
}

Keywords: local ventilation exhaust, extreme trajectories, discrete vortex method Abstract. The main problem of aspiration is the efficient localization of dust-and-gas emissions at the lowest energy consumption. One of the ways to reduce the energy consumption of the local open ventilation exhausts is their profiling by the found outline of the separated-flow region. The round local ventilation exhaust, made in the form of the bell, is the simplest and the most popular installation for catching dust-and-gas emissions. The purpose of this work is determining the influence of the expansion angle and the length of bell exhaust on the separated-flow region's characteristics, formed at the entrance to the bell, on the dynamics of dust particles and their collection efficiency. There was developed a mathematical model, computational algorithm and computer program for calculating separation flows at the entrance to round bell exhausts and for researching the dust particles behavior in their range of action.

The characteristic sizes of vortex regions at the entrance to the round bell exhaust have been determined. The analytic formula for calculating the separated-flow region at the entrance to the long round bell exhaust has been received. Profiling bells by the found outline of the separated-flow region allows improving the aerodynamic and acoustic properties of local ventilation exhausts. The extreme trajectories of dust particles of various sizes in the range of action of the round bell exhaust have been plotted. The efficiency criterion of a local ventilation exhaust in the form of the collection rate of a given dust particle, which amounts to the ratio of aspiration volume of an exhaust with bell and without it, has been suggested. The computational algorithms of calculating extreme trajectories of dust particles of the local ventilation bell exhaust's range of action are presented. The aspiration volumes and collection rates of dust particles of various fractions depending on the length and expansion angle of the bell are determined. A conclusion about the most efficient round bell exhaust is made. The characteristic sizes of separated-flow region at the entrance to bell outlet, located above a leakproof surface, have been determined. There was suggested an analytic formula for determining the separation line at the entrance to the bell with the expansion angle 180 degrees, located above a leakproof surface, profiling by which will allow to improve the aerodynamic and acoustic properties of a local ventilation exhaust. 


\section{INTRODUCTION}

Local venting suction socket is simplest and most spread element of exhaust ventilation systems. Interesting is determination of the boundaries of the gas separated flows area at inlets of the suction channels. Profiling on these boundaries improve the aerodynamic and acoustic properties of socket. Similar research for the slot-type suction-socket were first presented in a series of articles [1-3]. There used of complex variable theory methods. Separated flows for round-type suction socket investigated using the non-stationary discrete vortex method [4-5]. Characteristic dimensions of the vortex region inlet to the socket (Figure 1) at a fixed point in time were obtained. But it was not taken into account, that these dimensions pulsate with time. The paper [6] was an attempt to average over time value of the characteristic dimensions. Averaging was carried out by finding arithmetic mean of the values of 20 random points in time. The calculation was quite laborious. But 20 points in time is not sufficient to obtain convergent results in some cases. Therefore, it became necessary to calculate the gas separated flows area by the stationary discrete vortex model. Idea of this model was first described in the article [7], and then developed in the works [8-11,16]. Moreover, it is of interest determination such angles and length of suction socket, for which local venting suction socket more efficient.

\section{EFFICIENCY CRITERIA OF SUCTION SOCKET}

There are various approaches to determining of socket efficient. One of them is the criterion irregularity of suction which is determined by speed ratio at the center of the socket to the average velocity of suction $\eta=v_{c} / v_{0}$. For duct without the socket according to the data [12] $\eta=0,95-0,97$; for $\alpha=60^{\circ}-\eta=1,03 \div 1,09$; for $\alpha=90^{\circ}-\eta=1,65 \div 1,75$. The studies were conducted by Fialkovskaya, T.A. [13] and Baturin, V.V. [14]. It determines field of velocities in the zone of exhaust hood square and rectangular shape with aspect ratio of 1:2, $1: 3,1: 4$ and at different angles between defining lines from $50^{\circ}$ to $90^{\circ}$ (obviously it is the angle $\alpha$ shown in Figure 1).

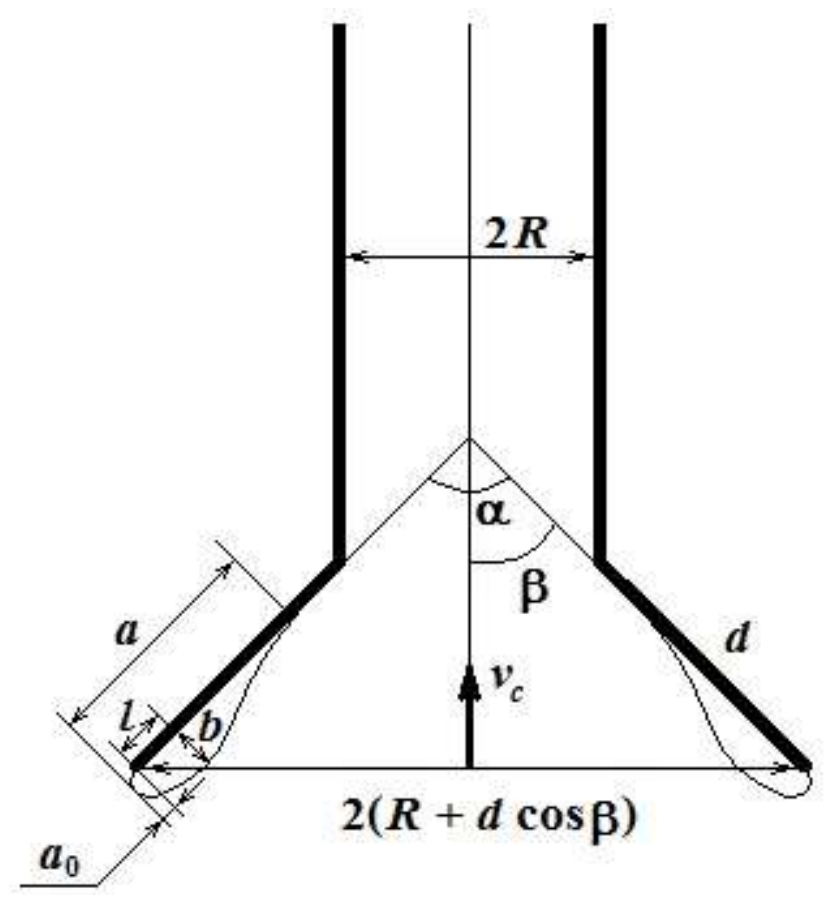

Figure 1: Round local ventilation suction - bell 
About the length of socket information is not accounted. About suctions of round-socket is also no mentioned. It was concluded, that because hoods with opening angle $\alpha=60^{\circ}$ the relative velocity is nearly to the average velocity in the center of hood hole the suction hole (flow rate per area), then the apex angle of hood must be less than $\alpha=60^{\circ}$. Dimensions of the inlet hood must be greater than dimensions of the heat source.

Further, approval about most efficient $\alpha=60^{\circ}$ published in numerous of books. For example in the book [15] it indicated that in order to hood working by full air intake cross section, opening angle of hood accept usually not more than $\alpha=60^{\circ}$. At larger angles actually acts only the central part of hood, and on the periphery appeared dead zones.

There are also other positions. It stated that the hood should be done with the opening angle $\beta=60^{\circ}$ in the Posohin V.N. book [15]. It was noted that in this case the dimension of the vortex area at the inlet of the socket is minimal and accordingly the "efficient dimension" hood is maximum. But here under the opening angle is meant $\beta=\alpha / 2$. The researches were conducted with the help of Conformal Mapping Method and [1-3] considered slot-socket suction. At here width of effective suction accepted as efficiency criterion.

Other efficiency criterion is highest rate of suction in the suction socket axis. It is shown by numerical and analytical studies [17] that the axial velocity reaches a maximum value when $\beta=\alpha / 2=90^{\circ}$. It used Conformal Mapping Method and discrete vortex Method too. Also it considered slot-type and round-type suction socket.

It can specify the aerodynamic drag as one more efficiency criterion of suction socket. By the graphs given in [18] we conclude, that the suction socket has smallest local resistance coefficient (1.r.c.) at $\alpha=50^{\circ}-100^{\circ}$. It depends on the length of the socket. If the socket length is smaller, then the change of 1.r.c. from the opening angle is approaching to linear law.

It proposed to introduce another criterion of efficiency: non-dimensional capture coefficient - the ratio of the aspiration volume of the suction-socket to aspiration volume of air duct without socket, because the local ventilation suckings [16, 19, 21-25] used not only for capture the excess of heat but also for various types of dust capture, which working with abrasive-metallic dust, wood dust, cement, sand, welding fumes and etc. Aspiration volume - is the volume of space being in which the dust particle with preset geometrical and physical properties will be caught by the local ventilation suction.

The main goal of the paper is the following: to elaborate a mathematical model and an algorithm of calculation of separation flow region at the inlet round-type suction socket with the use of the stationary discrete vortex method; to reveal the regularities in changes of the parameters of vortex region at socket inlet and to make a research of the efficiency of the local suction socket with the use of the suggested criterion.

\section{DEVELOPMENT OF A MATHEMATICAL MODEL AND A COMPUTATIONAL ALGORITHM}

In order to develop a mathematical model of the separated flow at the inlet to the round suction-bell used discrete infinitely thin vortex rings. Border suction funnel discretize attached set of vortex rings and control points (arbitrary points on a circle, embracing the suction-bell). The free surface of current begins on the sharp edge of the bell, the initial approximation is given for it. A discrete model in the meridian half-plane is shown in Figure 2 . The crosses are depicted from control points, in which fulfils the condition of impenetrability - velocity along the direction of the normal is zero. The shaded circles -It is the attached ring vortices. Hollow circles - free vortex rings. 


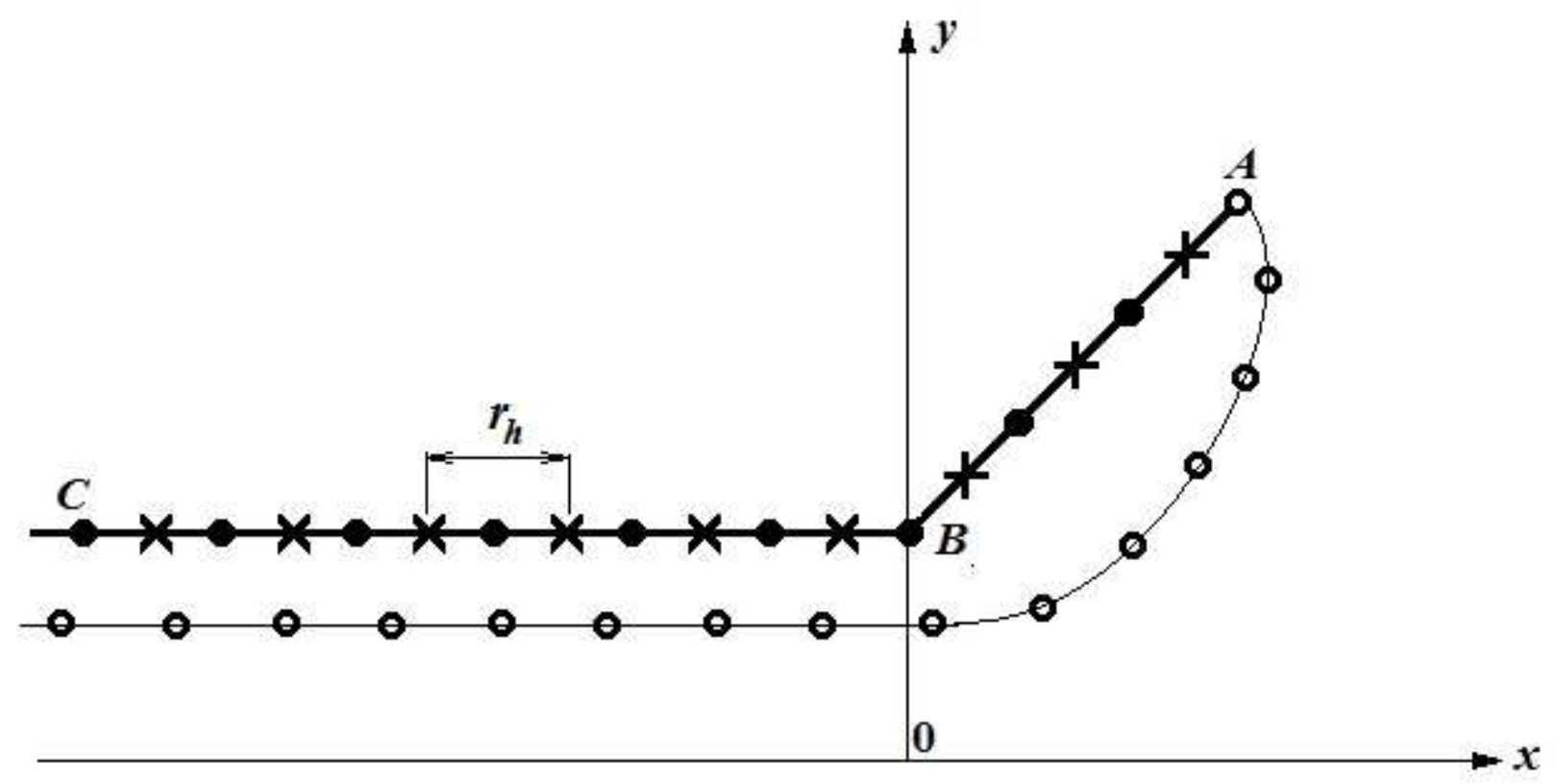

Figure 2: Discrete model of separated flow at the inlet to the suction-bell

Let $N$ - number of attached vortex rings ; $N_{s}$ - the number of free vortex rings; $x^{p}$ - control point, $p=1,2, \ldots, N$.

The velocity at any point $\mathrm{x}$ along the direction $\vec{n}$ is calculated using the formula:

$$
v_{n}(x)=\sum_{q=1}^{N} \Gamma\left(\xi^{q}\right) G\left(x, \xi^{q}\right)+\gamma \sum_{q=1}^{N_{s}} G\left(x, \zeta^{q}\right),
$$

Where $\xi^{q}$ - the location of $q$-th attached vertex ring with circulation $\Gamma\left(\xi^{q}\right), \gamma=$ const given free circulation of the vertex ring, $\zeta^{q}$ - location of the point $q$-th free vortex ring.

Function $G(x, \xi)$ expresses the influence of that point x of vortex ring with single loop, located at the point $\xi$

$$
\begin{aligned}
& \begin{cases}G(x, \xi)=\frac{\left(A_{1} b+A_{2} a\right)}{b} \cdot \frac{4}{(a-b) \sqrt{a+b}} E(t)-\frac{A_{2}}{b} \cdot \frac{4}{\sqrt{a+b}} F(t) & \text { at } b \neq 0, \\
G(x, \xi)=\frac{\xi_{2}^{2} \cdot n_{1}}{2 a \sqrt{a}} & \text { at } b=0,\end{cases} \\
& 2 x_{2} \xi_{2}=b>0, a=\left(x_{1}-\xi_{1}\right)^{2}+\xi_{2}^{2}+x_{2}^{2}>0, \quad A_{1}=\frac{\xi_{2}^{2} n_{1}}{4 \pi}, \quad A_{2}=\frac{\xi_{2}}{4 \pi}\left[\left(x_{1}-\xi_{1}\right) n_{2}-x_{2} n_{1}\right], \\
& F(t)=\int_{0}^{\pi / 2} \frac{d \theta}{\sqrt{1-t^{2} \sin ^{2} \theta}}, \quad E(t)=\int_{0}^{\pi / 2} \sqrt{1-t^{2} \sin ^{2} \theta} d \theta ; \\
& t=\frac{2 b}{a+b} ; F(t)=\sum_{i=0}^{4} c_{i}(1-t)^{i}+\sum_{i=0}^{4} d_{i}(1-t)^{i} \ln \frac{1}{1-t} ; E(t)=1+\sum_{i=1}^{4} c_{i}(1-t)^{i}+\sum_{i=1}^{4} d_{i}(1-t)^{i} \ln \frac{1}{1-t} ;
\end{aligned}
$$


If the distance from the point $\mathrm{x}$ to the point $\xi$ smaller than the steps of discreteness $r_{h}$, than this function is calcuated as follows:

$$
G(x, \xi)=\frac{\left(x_{1}-\xi_{1}\right) n_{2}-\left(x_{2}-\xi_{2}\right) n_{1}}{2 \pi r_{h}^{2}},
$$

where $r_{h}$-step of discreteness (distance between adjacent vortex rings).

In case $x=\xi$, function $G(x, \xi)=0$.

The numerical algorithm is constructed as follows. After specifying the location of the attached vortex points and the initial approximation for free vortices forms matrix.

$$
G^{p q}=G\left(x^{p}, \xi^{k}\right) ; p=1,2, \ldots, N ; q=1,2, \ldots, N .
$$

Further begins the first intergation.

Forms column of free terms:

$$
v^{p}=-\gamma \sum_{q=1}^{N_{s}} G\left(x^{p}, \zeta^{q}\right), p=1,2, \ldots, N .
$$

Solves systems of linear algebraic equations:

$$
\sum_{q=1}^{N} \Gamma^{q} G^{p q}=v^{p}, p=1,2,3, \ldots N
$$

From where determines unidentified quantities $\Gamma^{q}=\Gamma\left(\xi^{q}\right)$.

Constructed free current lines, starting with a sharp edge A. Using the formula (1), at $\vec{n}=\{1,0\}$ calculated velocity components $v_{x}$, at $\vec{n}=\{0,1\}$ calculated velocity componets $v_{y}$. Following point $\left(x^{\prime}, y^{\prime}\right)$ is determined from the previous $(x, y)$ using the given formula:

$$
x^{\prime}=x+\Delta t v_{x} / \sqrt{v_{x}^{2}+v_{y}^{2}}, \quad y^{\prime}=y+\Delta t v_{y} / \sqrt{v_{x}^{2}+v_{y}^{2}},
$$

where $\Delta t$ - step, which is chosen sufficiently small.

Integration process for building free surfaces of current finishes, if the position of the free surface of current stops to change with precisión to the desired accuracy.

After the parameters of separated flow región were determined( Figures 3-7).

Proposed formula for determining the boundary of the vortex at the inlet to the suctionbell.

$$
\bar{x}(t)=a \frac{1-e^{\frac{t-2 \pi-\beta}{3,1 t-3(\pi+\beta)}}}{R(t-\pi-\beta)^{b}} \cos t+\bar{d} \cos \beta ; \quad \bar{y}(t)=a \frac{1-e^{\frac{t-2 \pi-\beta}{3, t-3(\pi+\beta)}}}{R(t-\pi-\beta)^{b}} \sin t+1+\bar{d} \sin \beta
$$

where $\pi+\beta<t \leq 2 \pi+\beta ; \quad \bar{x}(t)=x(t) / R ; \quad \bar{y}(t)=y(t) / R$

$a=(0,03 \bar{d}-0,034) \frac{\beta}{\pi}+0,01 \bar{d}+0,032 ; \bar{d}=d / R ; \quad b=0,38-0,48 \frac{\beta}{\pi}$.

The formula is valid for long sockets, where $15^{\circ} \leq \beta \leq 90^{\circ}, l / B \geq 2$; when $l / B=1$ and $45^{\circ} \leq \beta \leq 90^{\circ}$. The calculation is carried out with $x>0$. 


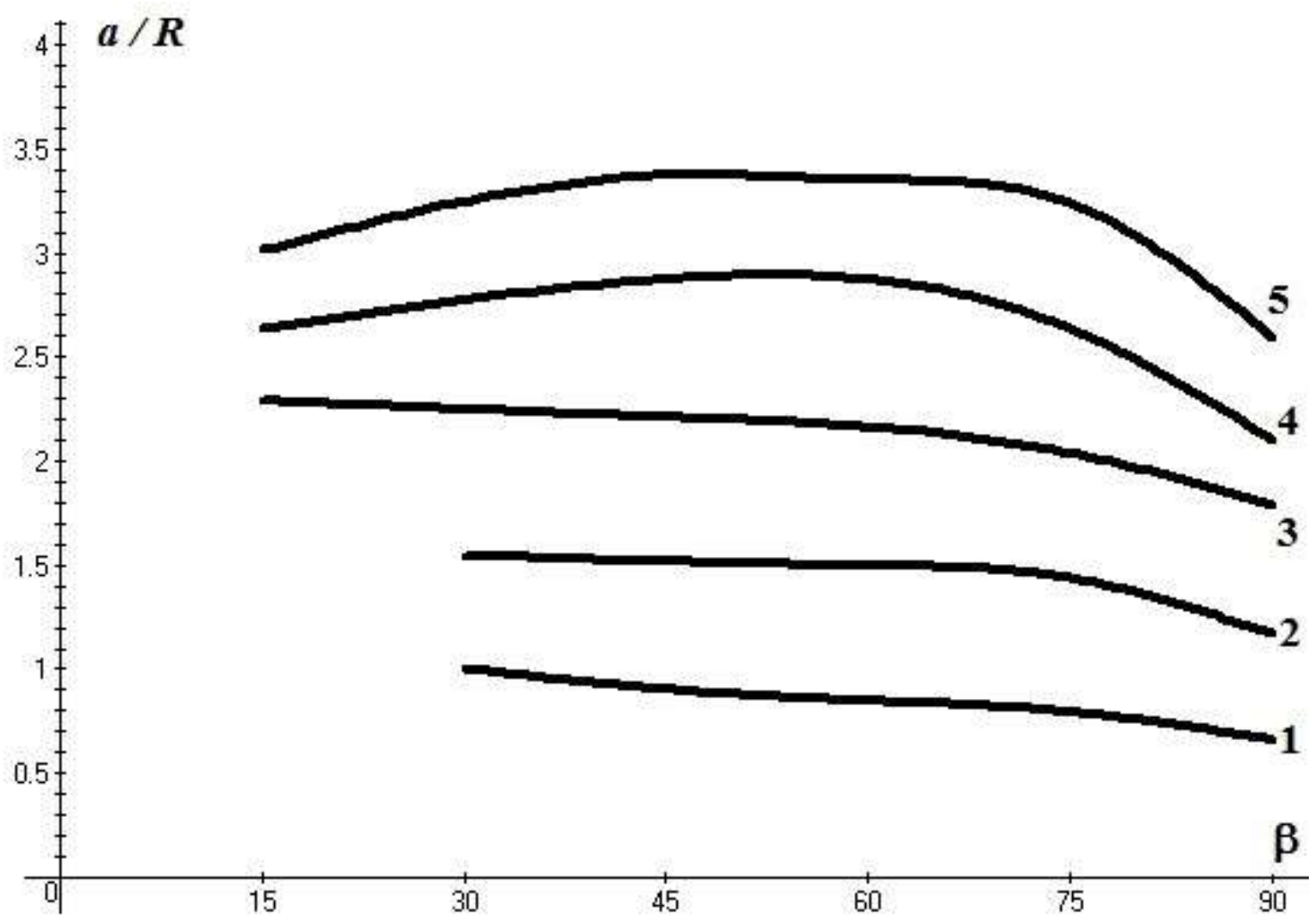

Figure 3: The dependence of the vortex field length $a$ at the entrance to the suction-bell

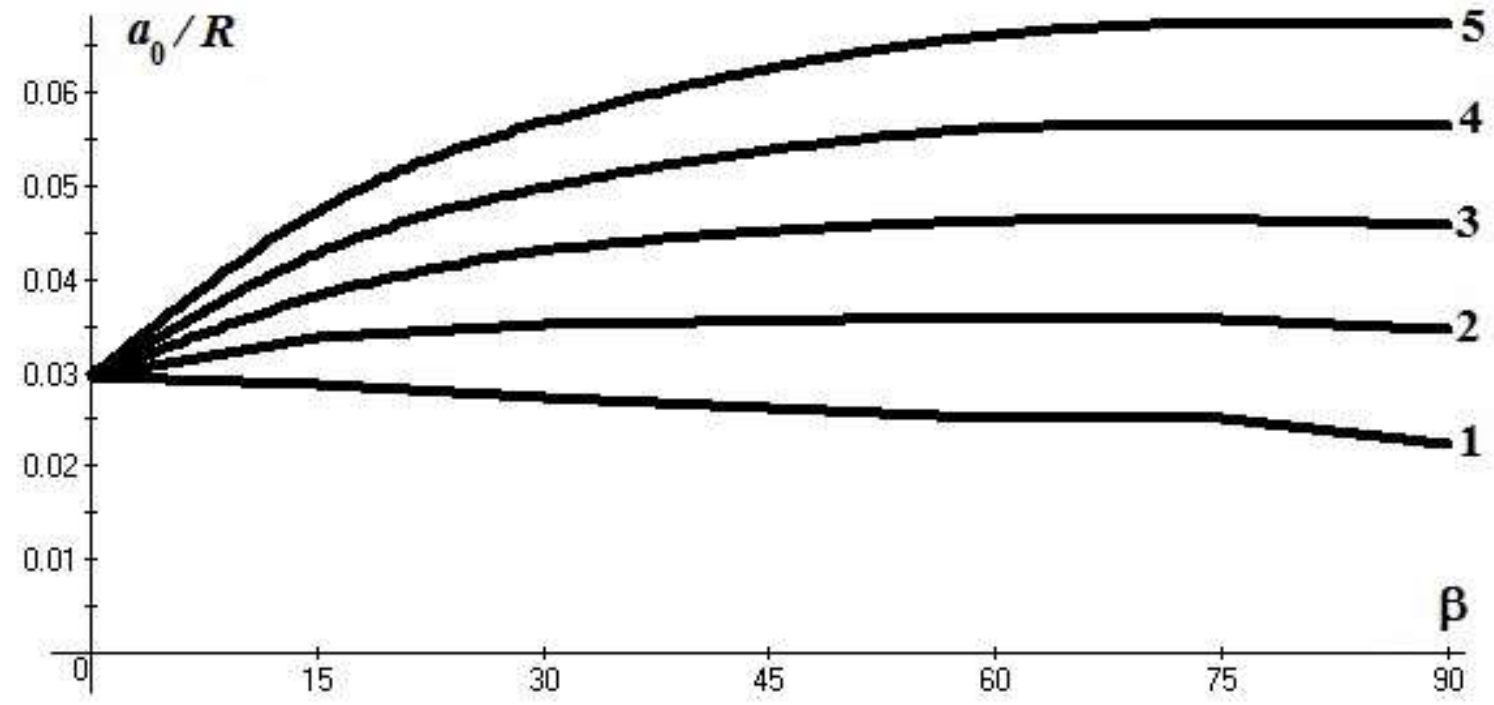

Figure 4: The change in the value $a_{0}$ of removal of the separation region behind the socket 


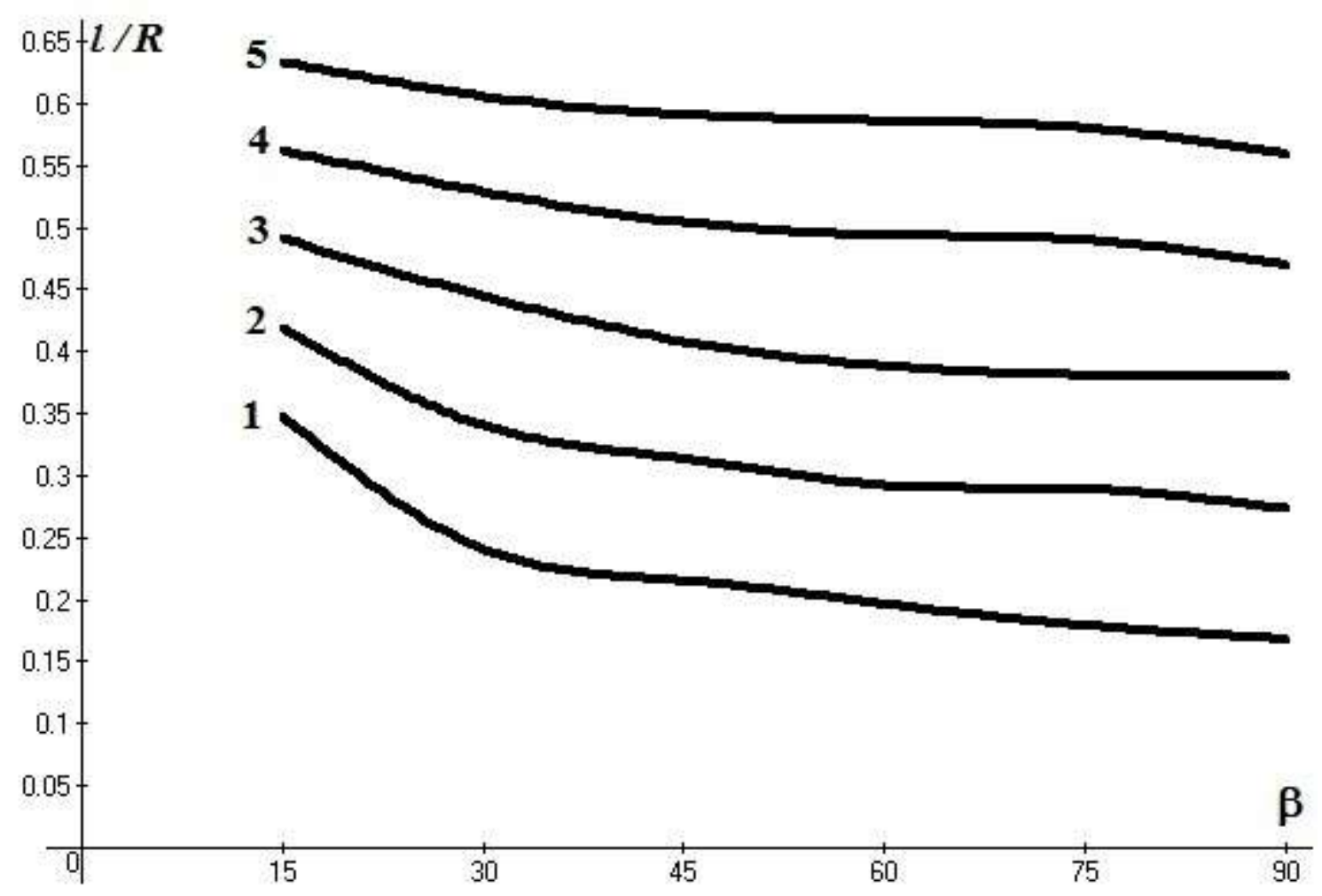

Figure 5: The change of parameter $l$ from the angle of the funnel opening

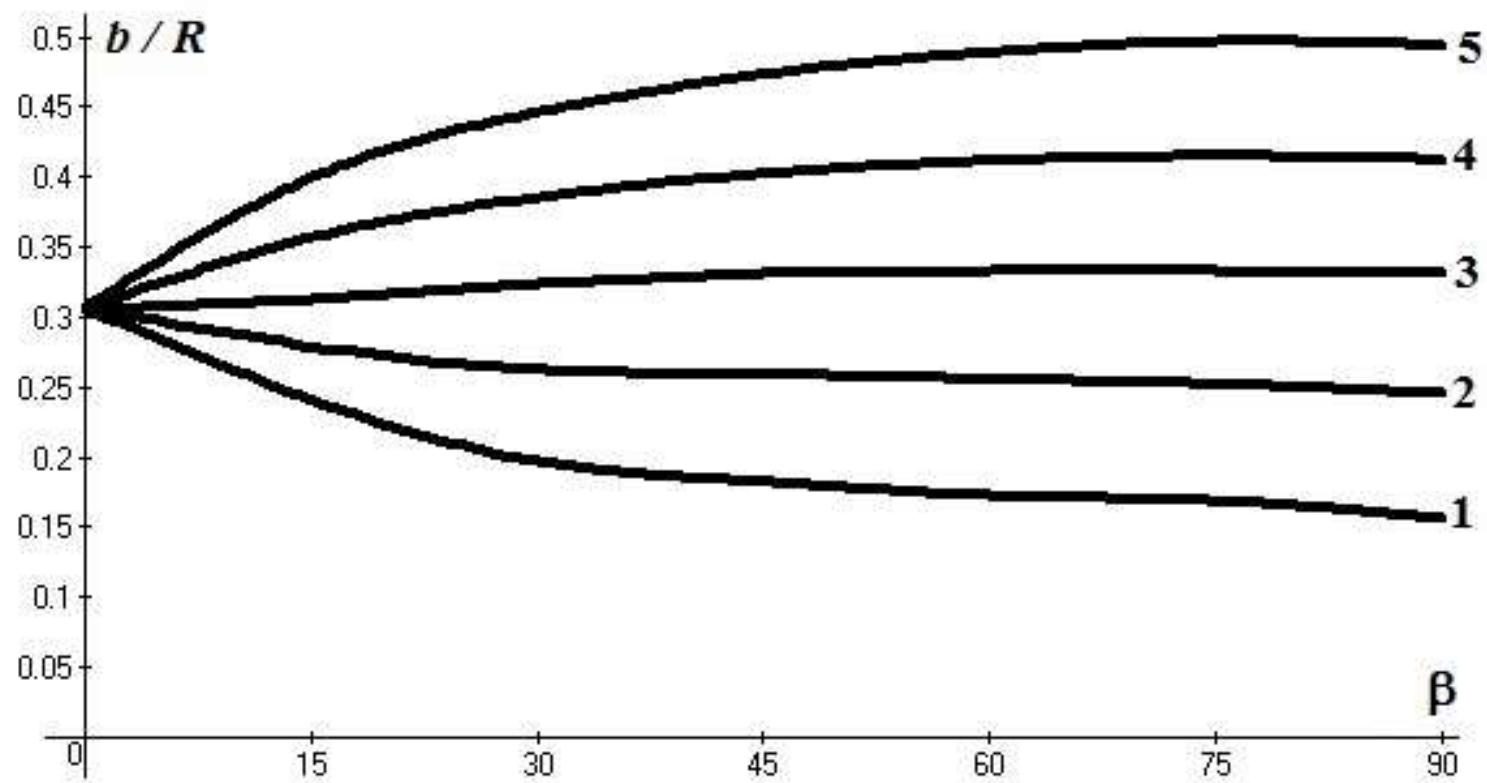

Figure 6: The change of the maximum thickness $b$ of separation region 


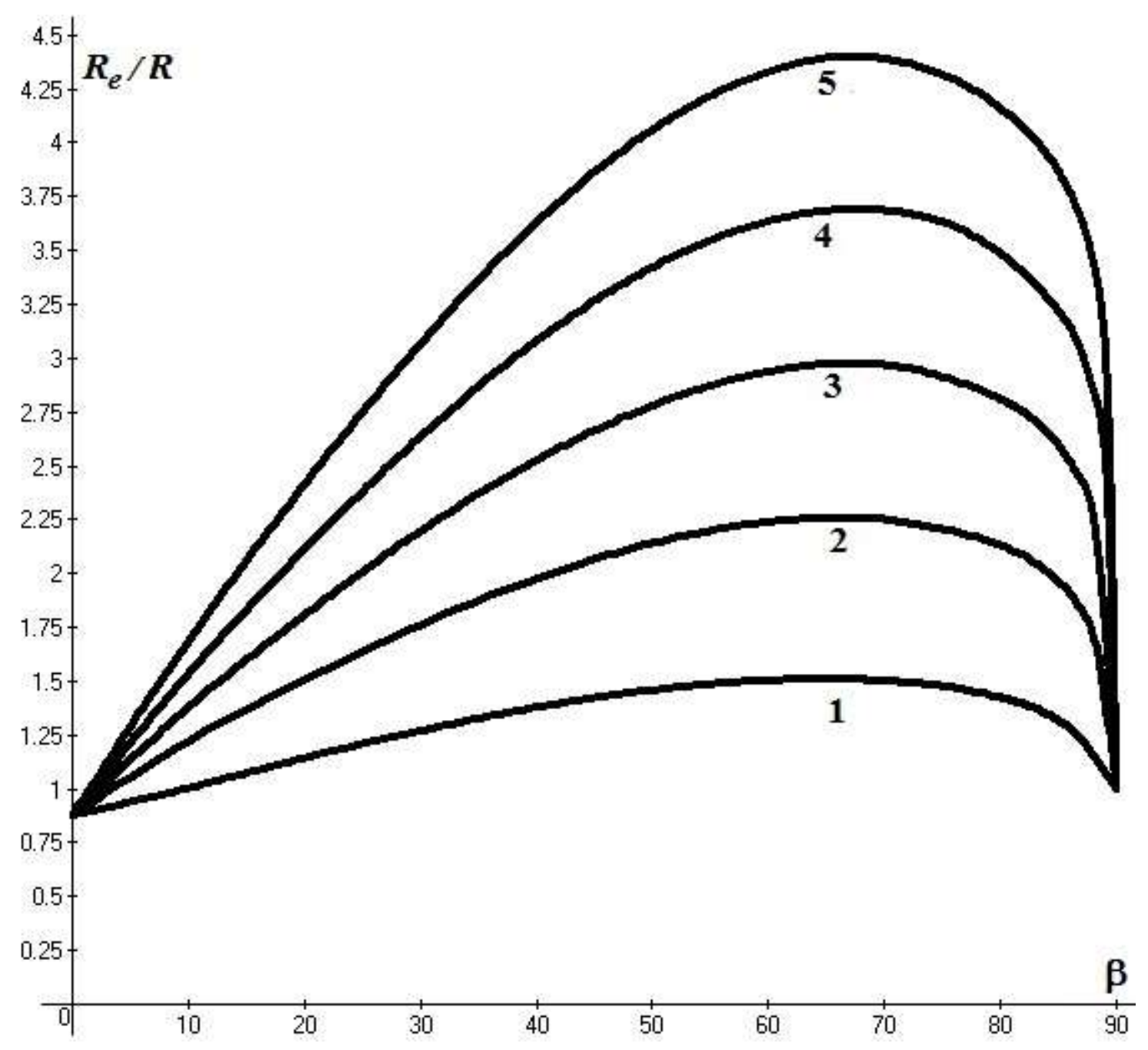

Figure 7: The change of radius of the effective absorption

\section{THE DYNAMICS OF DUST PARTICLES}

The trajectories of dust particles, build with the help of differential equation[17]:

$$
\frac{\pi d_{e}^{3}}{6} \rho_{p} \frac{d \vec{v}_{p}}{d t}=\frac{\pi d_{e}^{3}}{6} \rho_{p} \vec{g}-\psi \chi S_{m} \rho_{a} \frac{\left|\vec{v}_{p}-\vec{v}_{a}\right| \cdot\left(\vec{v}_{p}-\vec{v}_{a}\right)}{2},
$$

where $\vec{v}_{a}$ - velocity of the air; $\rho_{a}-$ air denstity; $\vec{v}_{p}-$ the particles velocity; $\rho_{p}$-the particles density; $d_{e}$ - equivalent diameter of the particles; $\vec{g}$ - accleration of free fall; $S_{m}=\pi d_{e}^{2} / 4-$ mid-section area of the particle; $\chi$ - coefficient of its dynamic form; $\psi$ - coefficient of air resistance:

$$
\psi=\left\{\begin{array}{l}
24 / \operatorname{Re}, \quad \text { at } \quad \operatorname{Re}<1, \\
24\left(1+1 / 6 \cdot \operatorname{Re}^{2 / 3}\right) / \operatorname{Re}, \text { at } \quad 1 \leq \operatorname{Re}<10^{3}, \\
24 / \operatorname{Re} \cdot\left(1+0,065 \operatorname{Re}^{2 / 3}\right)^{1.5}, \quad \text { at } \quad \operatorname{Re} \geq 10^{3},
\end{array}\right.
$$


where $\operatorname{Re}=\frac{\rho_{a}\left|\vec{v}_{p}-\vec{v}_{a}\right| d_{e}}{\mu}, \mu-$ coefficient of dynamic viscosity of air.

Limited trajectories of the dust particles have diameter 1-120 microns (Figure 8).

Volume of aspiration- It is the volume between the plane A-A (Figure 8), passing through the entrance into the bell and the surface of limited trajectory of dust particles.

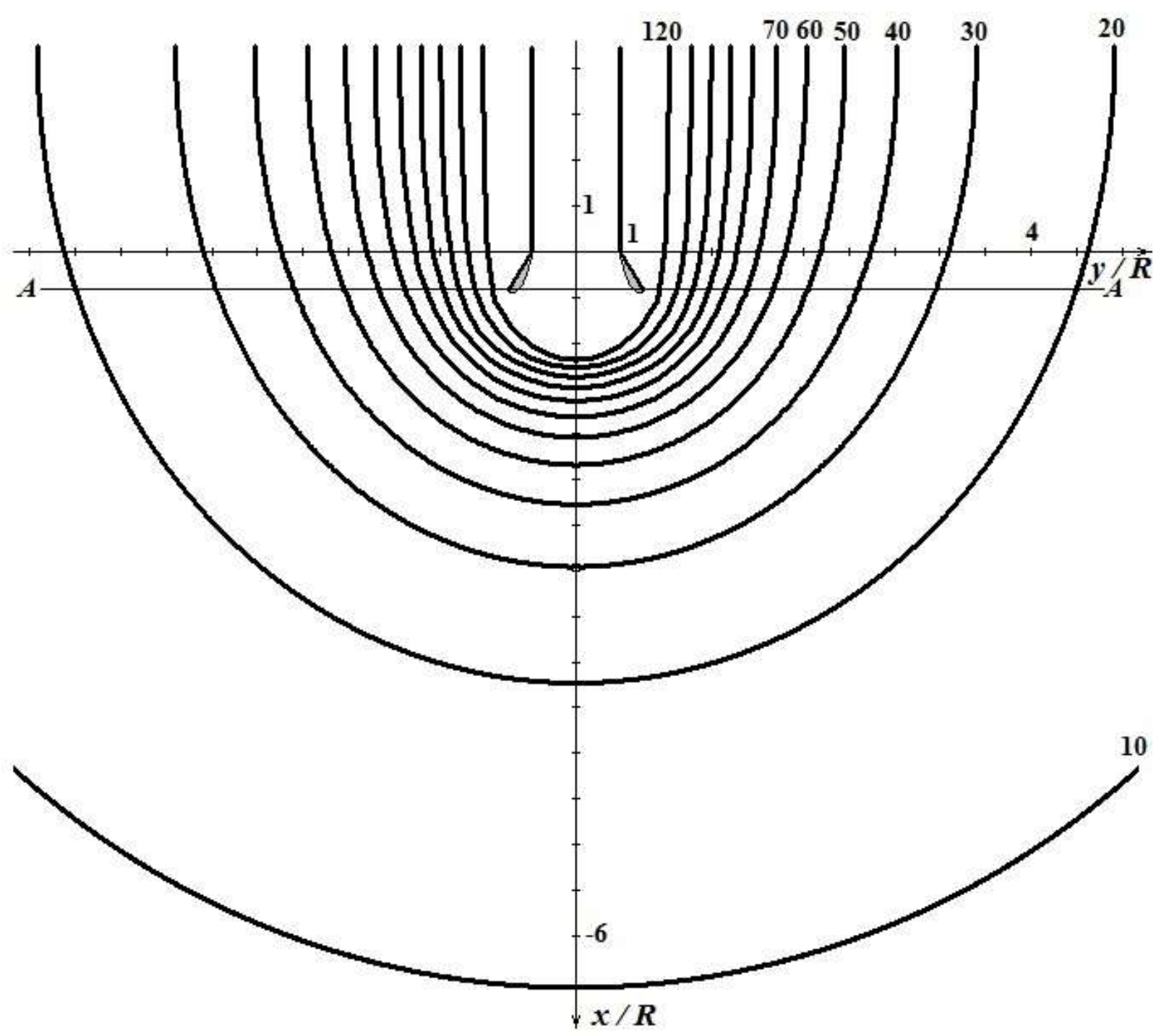

Figure 8: The limit trajectory of dust particles in the spectrum of action circular suction-bell length of 1gauge with an opening angle $\alpha=60^{\circ}$ when the middle suction speed is $10 \mathrm{~m} / \mathrm{s}$

Under the criterion of efficiency of local ventilation of suction-bell for dust particles, we understand the collection coefficient.

$$
k=V_{a} / V_{0},
$$

$V_{0}$ - volume of aspiration suction with the socket; $V_{0}$ - volume of the aspiration suction without socket. 
In calculations: $\rho_{p}=2500 \mathrm{~kg} / \mathrm{m}^{3} ; \chi=1 ; g=9,81 \mathrm{~m} / \mathrm{s}^{2} ; \rho_{a}=1,2 \mathrm{~kg} / \mathrm{m}^{3} ; v=1,5 \cdot 10^{-5} \mathrm{~m}^{2} / \mathrm{s} ;$ $v_{0}=10 \mathrm{~m} / \mathrm{s}$.

For particles with diameters $20 \mathrm{mkm}, 40 \mathrm{mkm}, 60 \mathrm{mkm}, 80 \mathrm{mkm}$ the collection coefficient is highest when $\beta=90^{\circ}$ (Figure9).

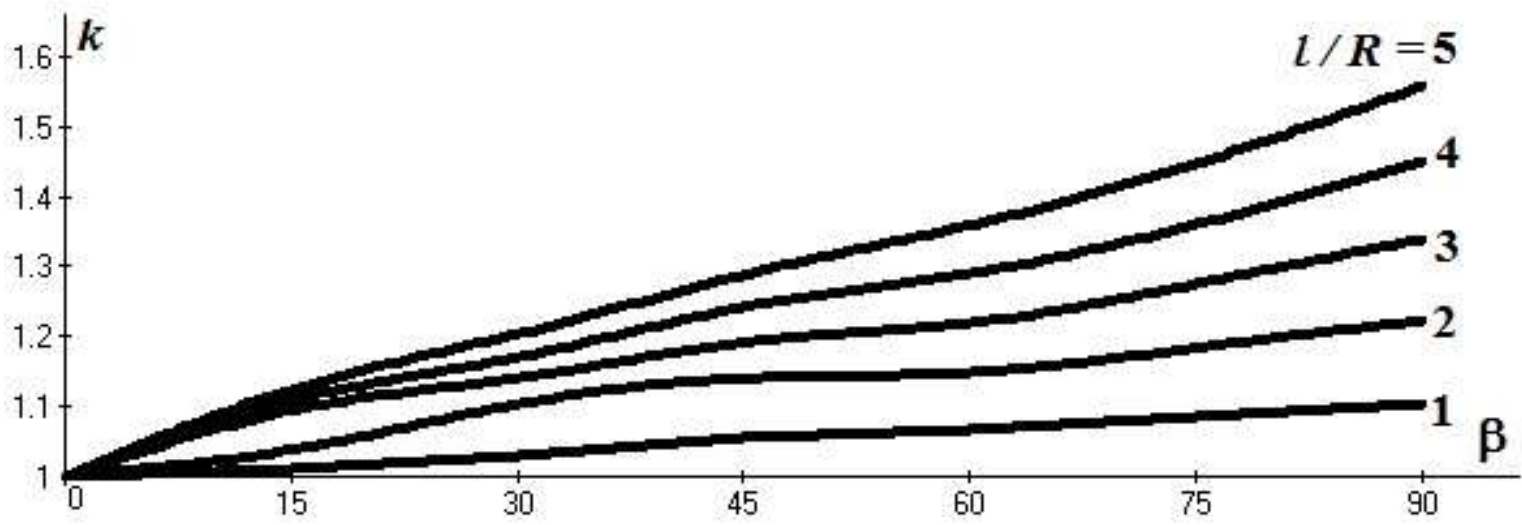

a) $20 \mathrm{mkm}$

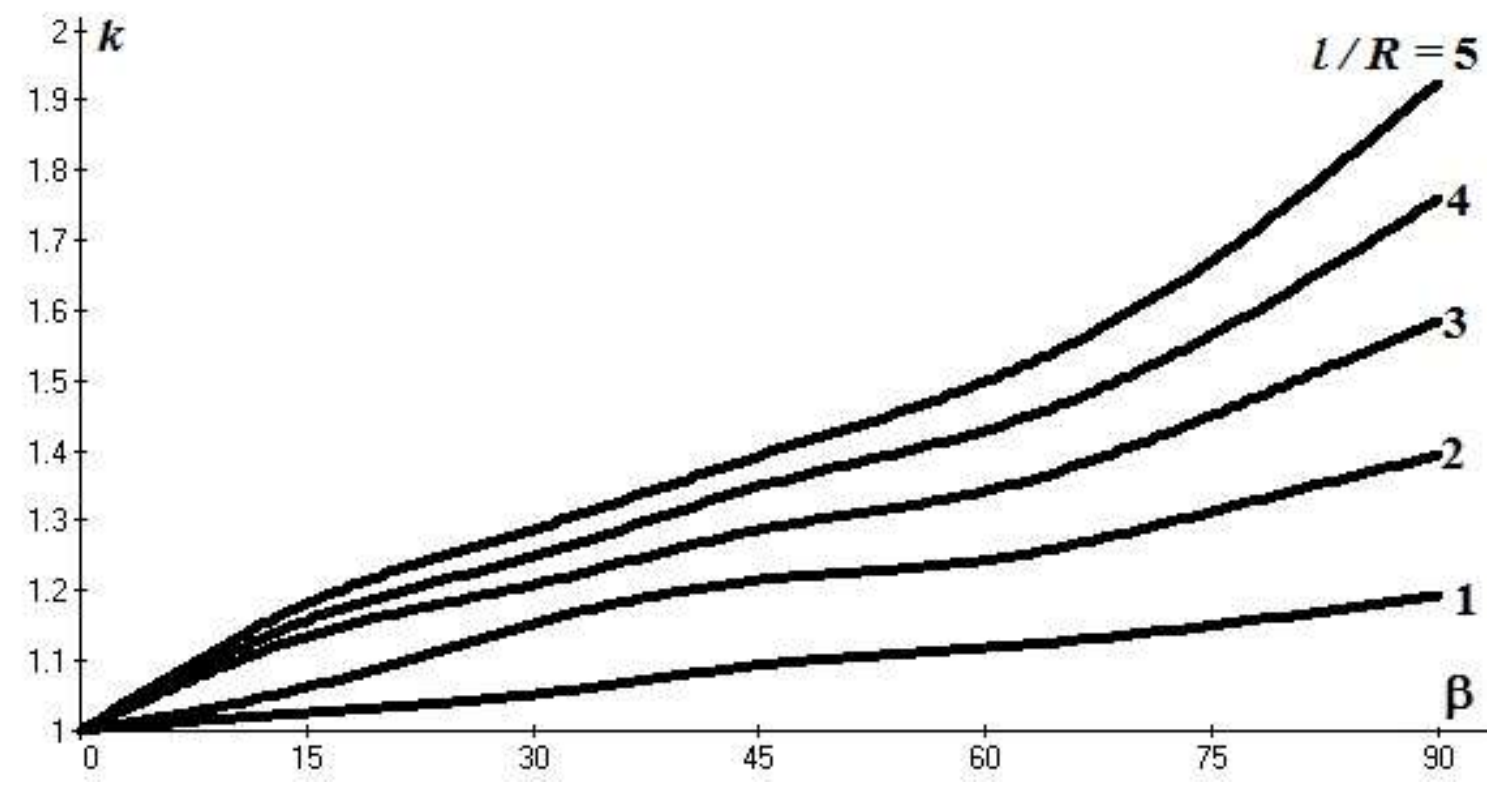

b) $40 \mathrm{mkm}$ 


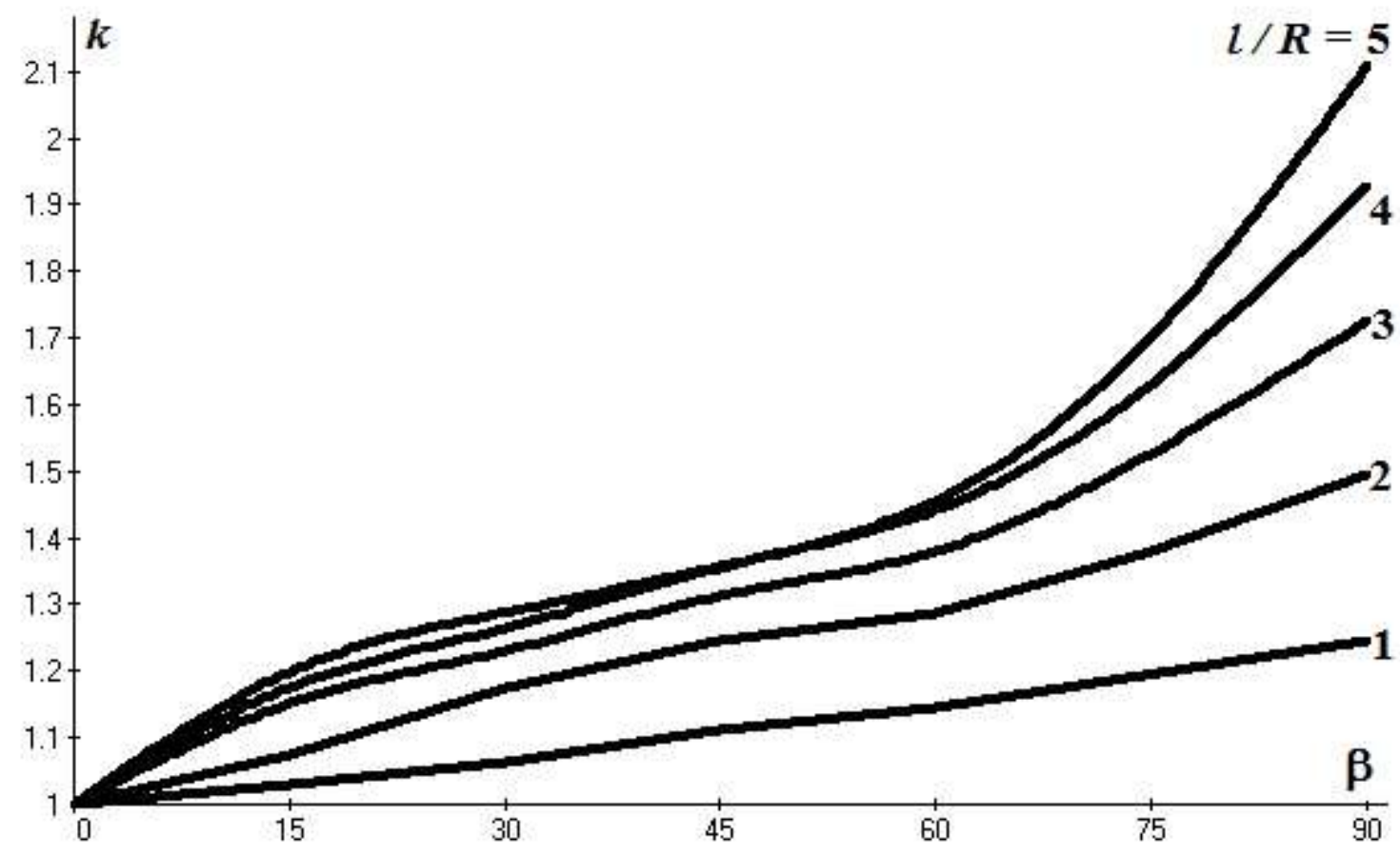

c) $60 \mathrm{mkm}$

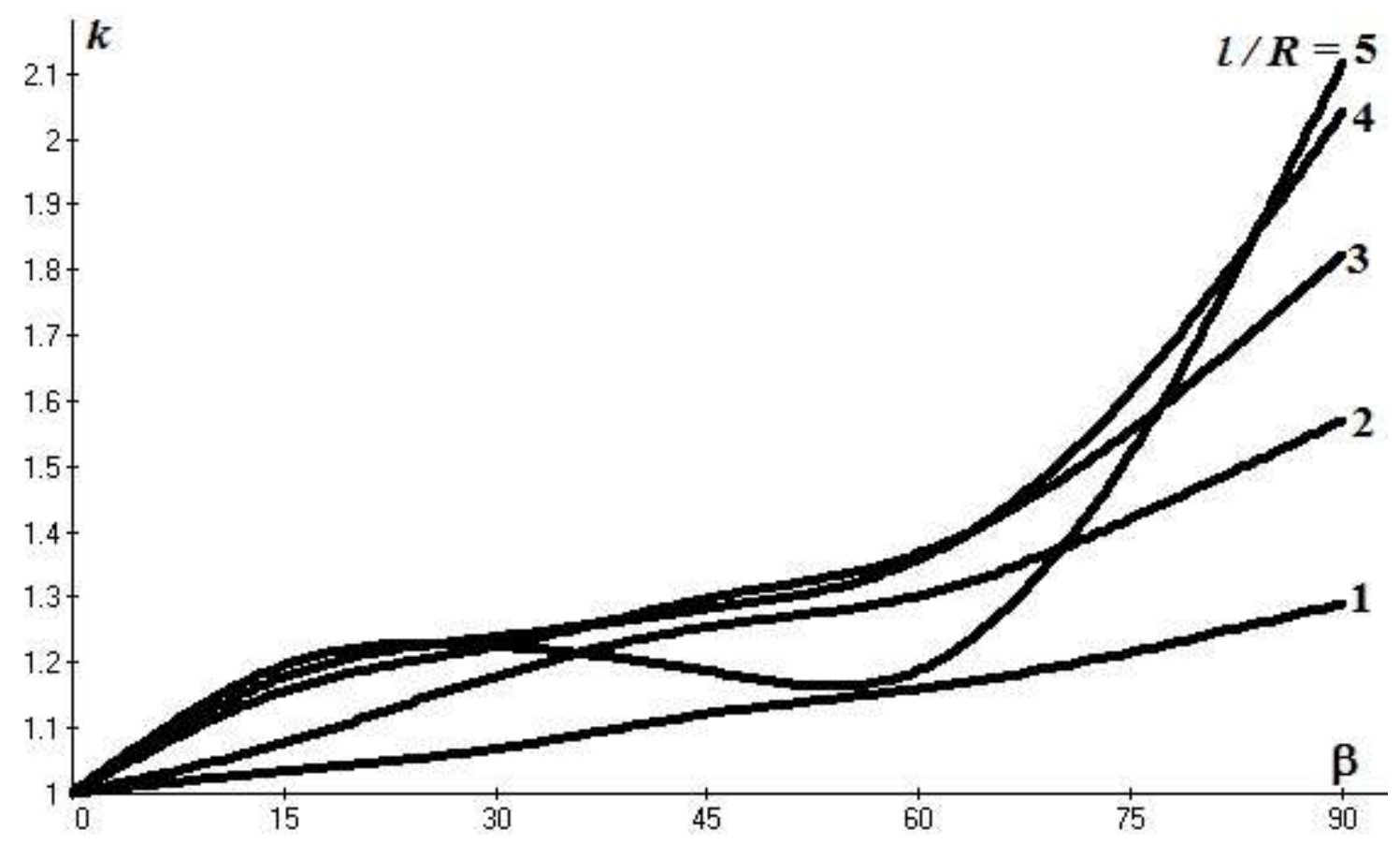

d) $80 \mathrm{mkm}$

Figure 9: The change in the coefficient capture from the angle for different lengths of the funnel The effect of collection coefficient on the length of the socket is shown in figure 10 . 


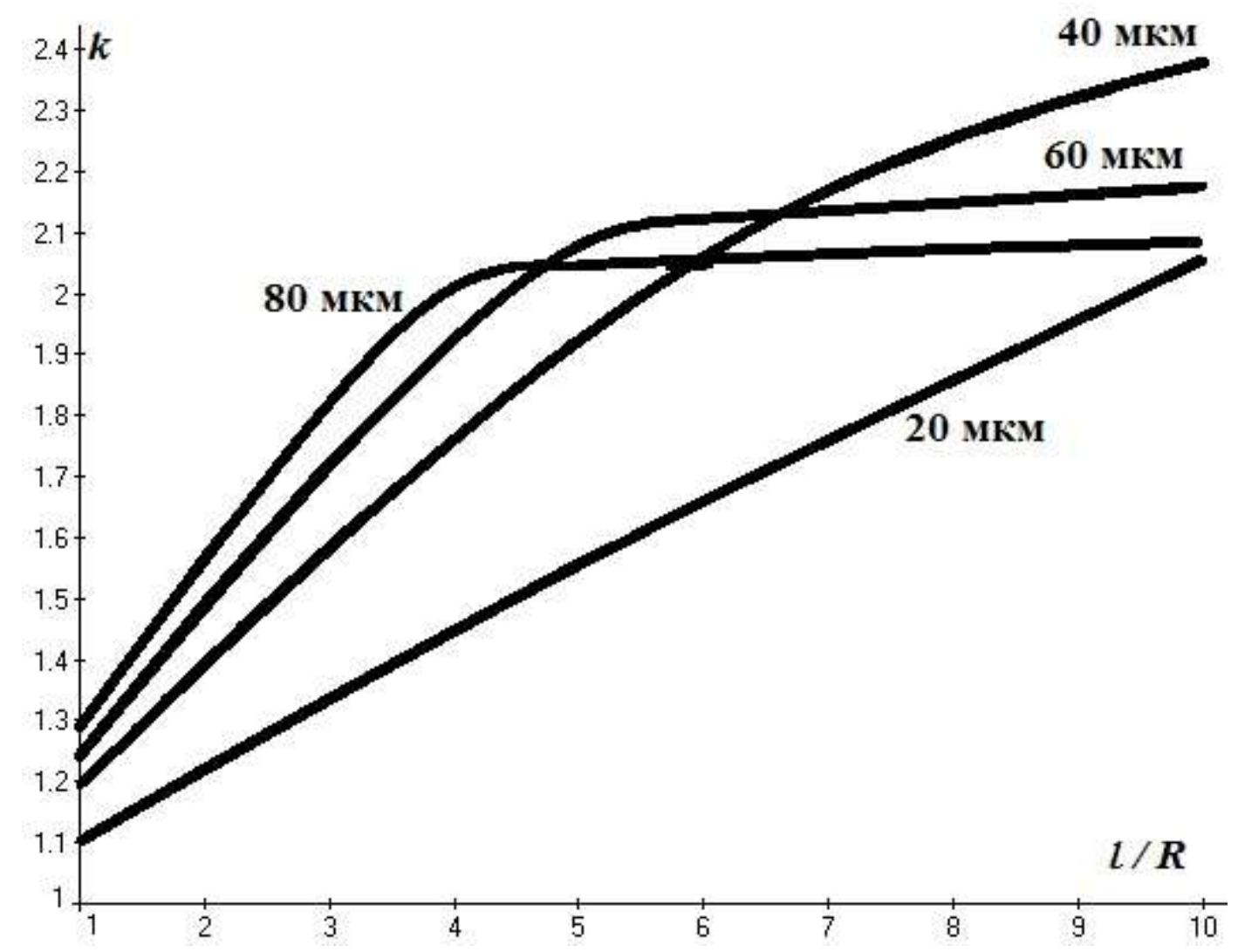

Figure 10: The change in coefficient capture according to the length of the socket when $\beta=90^{\circ}$.

\section{CONCLUSIONS}

- A mathematical model is designed, computational algorithm and a computer program to calculate separated flows at the entrance to the round suction-bells and to study the behavior of dust particles in the spectrum of their activities.

- Identified characteristic dimensions of the vortex areas at the entrance to the round suction-bell. Found an analytical formula for calculating the boundaries of the separation region at the entrance to a long suction-bell. Profiling sockets found on the outline of the tear region will improve the aerodynamic and acoustic properties of the local air suction.

- Built limiting trajectories of dust particles of different sizes in the range of action of the round suction-bell. Proposed the criterion on the effectiveness of local suction ventilation in the form of collection coefficient of the given dust particles, which is equal to the ratio of volumes of aspiration suction with socket and without it.

- The most effective suction is the funnel with an opening angle $\alpha=180^{\circ}$ and length of at least 4 gauge.

\section{ACKNOWLEDGEMENTS}

The reported study was funded by RFBR according to the research project № 16-0800074a и and Russian President Scholarship СП-121.2016.1. 


\section{REFERENCES}

[1] V.N. Posokhin, N.B. Salimov, K.I. Logachev, A.M. Zhivov, Regarding analysis of flows around a slotted bell-shaped hood inlet. Message 1. News of Higher Educational Institutions. Construction, 8, 70-76, 2002

[2] V.N. Posokhin, N.B. Salimov, K.I. Logachev, A.M. Zhivov, Regarding analysis of flows around a slotted bell-shaped hood inlet. Message 2. News of Higher Educational Institutions. Construction, 9, 80-85, 2002

[3] V.N. Posokhin, N.B. Salimov, K.I. Logachev, A.M. Zhivov, Regarding analysis of flows around a slotted bell-shaped hood inlet. Message 2. News of Higher Educational Institutions. Construction, 10, 81-85, 2002.

[4] K.I. Logachev, A.I. Puzanok, V.N. Posokhin, Analysis of flows around a slotted bellshaped hood inlets using the discrete vortex method. Izv. vuzov. Problems of energetics, 7-8, 61-69, 2004.

[5] K.I. Logachev, V.N. Posokhin, A.I.Puzanok, Geometrical characteristics of flows at the entrance of hood-shaped exhausts. Engineering systems. AVOK North-West, 1, $12-$ 14, 2005.

[6] O.A. Averkova, A.E. Kanar, E.I. Tolmacheva, I.V. Khodakov, Average characteristics of eddy regions at the entrance of a round bell duct. The book includes: Energy saving, information technologies and sustainable development. Electronic scientific publications: digest of the International research and application internet conference. FSBEI "IzhGTU named after M.T. Kalashnikov", Izhevsk, 162-167, 2014

[7] K.I. Logachev, V.N. Posokhin, Analysis of flows around intake pipes of round crosssection. Izv. vuzov. Aeronautical engineering, 1, 29-32, 2004.

[8] O.A. Averkova, I.N. Logachev, K.I. Logachev, Modeling of potential flows with unknown boundaries on the basis of stationary discrete vortices. Computational methods and programming, 12, 213-219, 2011.

[9] O.A. Averkova, I.N. Logachev, K.I. Logachev, Modeling of flow separation at the inlet of a suction channel in regions with cuts. Computational methods and programming, 13, 298-306, 2012.

[10] O.A. Averkova, I.N. Logachev, K.I. Logachev, Separation flows in the exhaust ducts spectra. Moscow-Izhevsk: IKI, 2012.

[11] I.N. Logachev, K.I. Logachev, O.A. Averkova. Local Exhaust Ventilation: Aerodynamic Processes and Calculations of Dust Emissions. Boca Raton: CRC Press, 2015.

[12] P.N. Kamenev. Heating and ventilation. Part 2. Ventilation. Moscow: State publishing house of building, architecture and building materials literature. - 1959.

[13] T. A. Fialkovskaya, Exhaust hoods and fume chambers. Moscow: Stroyizdat, 1947.

[14] V. V. Baturin, The fundamentals of industrial ventilation. Moscow: Profizdat, 1990.

[15] E.A. Shtokman, V.A. Shilov, E.E. Novgorodsky, I.I. Savvidi, T.A. Skorik, V.V. Pashkov, Ventilation, conditioning and air purification at food industry enterprises. Ed. by E.A.Shtokman. Moscow: ASV publishing house, 2001.

[16] V. N. Posokhin. Ventilation aerodynamics. Moscow: AVOK-PRESS, 2008. 
[17] I.N. Logachev, K.I. Logachev, O.A. Averkova. Local Exhaust Ventilation: Aerodynamic Processes and Calculations of Dust Emissions. Boca Raton: CRC Press, 2015.

[18] I.E. Idelchik. Hydraulic resistance guide book. Moscow: Mashinostroeniye, 1977.

[19] V.G. Shaptala, V.V. Shaptala, A.V. Gavrilenko, Numerical simulation of cement-air mixture in the pneumochamber pump. Bulletin of BSTU named after V.G. Shukhov, 2, 159-161, 2015.

[20] Special functions reference book. ed. by M.Abramovits and I.Stigan. M.:Nauka, 1979.

[21] V.V. Shaptala, Modeling and calculation systems of cleaning dust emissions of cement production. Bulletin of BSTU named after V.G. Shukhov, 6, 205-209, 2015.

[22] V.G. Shaptala, V.V. Shaptala, The method of calculating efficiency of centrifugal collectors sticky dust in the production of building materials. Bulletin of BSTU named after V.G. Shukhov, 3, 58-62, 2014.

[23] V.A.Minko, Dust control of construction materials production processes. Voronezh: VSU Publishing, 1981.

[24] I.V. Khodakov, Modeling of detached flow at the entry polygonal suction hole. Bulletin of BSTU named after V.G. Shukhov, 2, 10-14, 2016.

[25] I.V. Khodakov, Numerical and experimental research of detached flow at the inlet in the suction duct with the mechanical screen. Bulletin of BSTU named after V.G. Shukhov, 3, 6-12, 2016. 